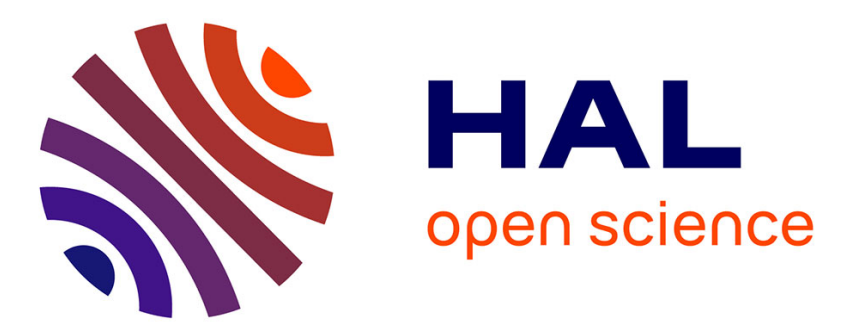

\title{
Atomic force microscopy-based single-cell indentation: Experimentation and finite element simulation
}

Hamid Ladjal, Jean-Luc Hanus, Anand Pillarisetti, Carol Keefer, A. Ferreira, Jaydev Desai

\section{- To cite this version:}

Hamid Ladjal, Jean-Luc Hanus, Anand Pillarisetti, Carol Keefer, A. Ferreira, et al.. Atomic force microscopy-based single-cell indentation: Experimentation and finite element simulation. IEEE/RSJ International Conference on Intelligent Robots and Systems., Oct 2009, St. Louis, MO, United States. pp.1326 - 1332, 10.1109/IROS.2009.5354351 . hal-00653118

\section{HAL Id: hal-00653118 https://hal.science/hal-00653118}

Submitted on 17 Dec 2011

HAL is a multi-disciplinary open access archive for the deposit and dissemination of scientific research documents, whether they are published or not. The documents may come from teaching and research institutions in France or abroad, or from public or private research centers.
L'archive ouverte pluridisciplinaire HAL, est destinée au dépôt et à la diffusion de documents scientifiques de niveau recherche, publiés ou non, émanant des établissements d'enseignement et de recherche français ou étrangers, des laboratoires publics ou privés. 


\title{
Atomic Force Microscopy-Based Single-Cell Indentation: Experimentation and Finite Element Simulation
}

\author{
Hamid Ladjal, Jean-Luc Hanus, Anand Pillarisetti, Carol Keefer, Antoine Ferreira and Jaydev P. Desai
}

\begin{abstract}
In order to understand and characterize the mechanical property and response of the mouse embryonic stem cells (mESC), we used the atomic force microscope (AFM) combined with the a PHANToM haptic feedback device. Atomic force microscopy has rapidly become a valuable tool for quantifying the biophysical properties of single cells or a collection of cells through force measurements. We report herein the mechanical characterization of single mESC indentationrelaxation measurements using micro-sphere AFM probes for fixed and live undifferentiated mESC. During cell indentation for both live cells and fixed cells undifferentiated, we provided force feedback to the user in real-time through the PHANToM haptic feedback device as the AFM tip was deforming the cell. The interface allowed the user to feel the cell indentation force in real time. The force was amplified for the human operator to perceive the change in force during cell indentation by the AFM cantilever. This information can be used as a mechanical marker to characterize state of the cell(live and fixed). As the interpretation of atomic force microscopy-based indentation tests is highly dependent on the use of an appropriate theoretical model of the testing configuration, a various contact models are presented to predict the mechanical behavior of an individual mouse embryonic stem cell(mESC)in different states. A comparison study with finite element simulations (FEM) of spherical tip indentation demonstrates the effectiveness of our computational model to predict the mESC deformation during indentation and relaxation nanomanipulation tasks.
\end{abstract}

\section{INTRODUCTION}

The realization of promising potential of stem cells for engineering bone, cartilage, muscle and other connective tissues requires a proper characterization of their unique biological, biochemical, proteomic, and biomechanical properties that are yet to be fully elucidated. The mechanical properties such as elasticity, membrane tension, cell shape, and adhesion strength may play an important role in the cell fate and differentiation [1],[2],[3]. The mechanical properties of biological cells have been studied with different techniques [4], the most popular are optical tweezers [5], magnetic beads [6], and micropipette aspiration [7]. However, those methods cannot compete with the precision that can be attained with Atomic force microscopy (AFM) method [8]. AFM has been widely used in the study of micro- and nanostructures including living cells. Modern AFM techniques allow solving a number of problems of cell biomechanics by simultaneous evaluation of the local mechanical properties and the topography of the living cells, at a high spatial

H. Ladjal, J-L Hanus and A. Ferreira are with Institut PRISME, ENSI de Bourges 88, Boulevrad Lahitolle, France antoine. ferreira@ensi-bourges. fr

A. Pillarisetti, C. Keefer, J.P. Desai are with the Department of Mechanical Engineering, University of Maryland, Maryland, USA jaydev@umd.edu

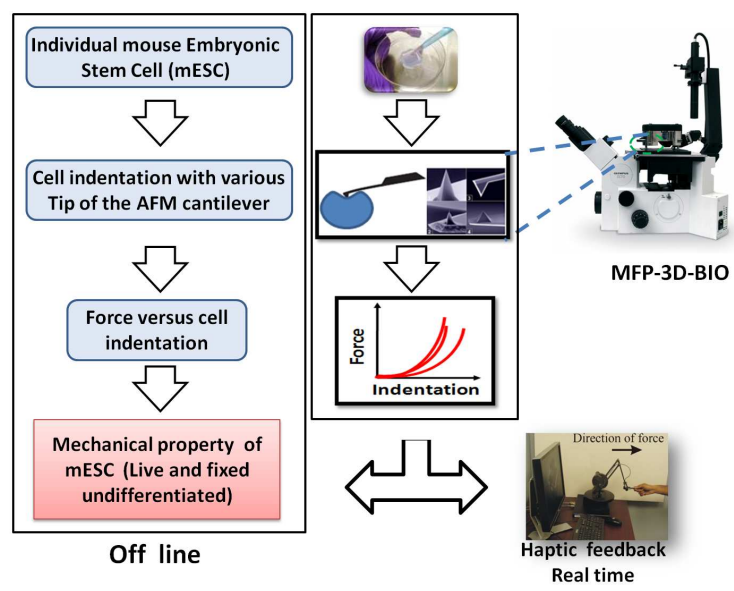

Fig. 1. Schematic showing the haptics-enabled Atomic Force Microscopy: The AFM is used to obtain fore and indentation data.

resolution and force sensitivity [9]. In these experiments AFM cantilever serves as a microindenter to probe the cell, and further analysis of force-indentation data yields the local Young's modulus. In addition, AFM indentation technique can be used to characterize the viscoelastic behavior of the cell cytoskeleton [10], including viscosity [11], loss and storage moduli [12], and stress relaxation times. AFMbased indentation techniques with force feedback have been applied here to characterize single-cell mouse embryonic stem cells (mESCs) [13]. We report herein the mechanical characterization of single mESC indentation-relaxation for different colonies of fixed and live undifferentiated mESCs. The proposed indentation tests use microsphere-modified AFM probes in order to estimate global elastic modulus for the cell to reflect the true global response of a mESCs[14], [15]. Distribution of the indenting load over several $\mu m^{2}$ area, averages the contribution of multiple cytoskeleton fibers and makes the contact analysis more accurate. Indeed, the use of $5 \mu \mathrm{m}$ diameter spherical indenter allows to reduce experimental elastic data dispersion several-fold compared to regular pyramidal sharp SiNi AFM tips [16], [17]. As the interpretation of AFM-based indentation tests is highly dependent on the use of an appropriate theoretical model of the testing configuration, our results demonstrate the applicability of the Hertz contact model as the most appropriate model compared to the capsule model or the JKR and DMT models. We have used the Hertzian contact model for the spherical tip indentor to compute the Young's modulus of the cells(live and fixed). We have demonstrated that even if the two dimensional mechanical models of mESCs are suitable 
for analytical studies, it gives a valuable understanding of the principles governing mechanical interactions within a cell. A numerical validation study of the Hertzian contact model has been conducted through a realistic two-dimensional finite element modeling (FEM). The numerical model is based on viscoelastic approximation with large deformations of cellular soft matter with material properties statically given for the time span of cell micromanipulation. Finally, the FEM simulations validate the mESC stress relaxation properties (hysteresis was about 15-20 percent) indicating that energy dissipation due to the material viscosity contribution is low at this probe velocity and force measurements are dominated by the cell elastic behavior. The proposed FEM model constitutes a basis for simulation of micro/nano-injection tasks using virtual haptic feedback model in tissue engineering and cancer research [18]. The paper consists of five sections. In Section II, we present the materials and methods used in our work. In Section III, we present the results of mouse embryonic stem cells characterization. In Section IV, we made a comparative study between the different analytical and numerical models. Finally, in Section V, we make some concluding remarks and give the direction for future work.

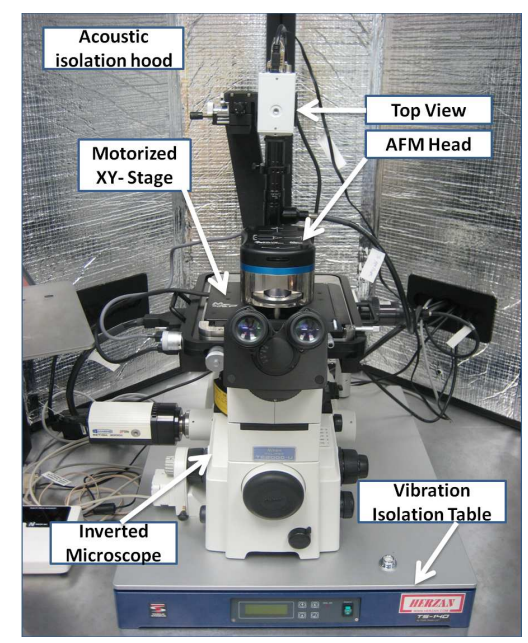

Fig. 2. Atomic Force Microscopy: The experimental set up for mESC indentation studies.

\section{EXPERIMENTAL SETUP}

\section{A. Description}

The nanoindentation of the cells was performed using the Atomic Force Microscope (Model: MFP-3D-BIO, Asylum Research, Santa Barbara, CA) combined with PHANToM haptic feedback device (Sensable Technologies, Inc.). The AFM is integrated with a top view module and mounted on a active vibration isolation table manufactured by Herzan (Laguna Hills, CA) as shown in Fig. 2. The top view module enables viewing of cells and easy alignment of the laser beam on the AFM cantilever. The XY stage (manual) allows the user to position the cell beneath the cantilever tip of AFM. The entire AFM set up is enclosed in a acoustic isolation chamber to prevent acoustic noise from interfering with the AFM measurements. The $x$ - and $y$-axes range of the scan head is $90 \mu \mathrm{m}$. The $z$-axis scan range is
$40 \mu \mathrm{m}$. The Atomic Force Microscopy (AFM) system is used to obtain force and cell deformation data from biological samples. The cantilever is moved by the piezoelectric scanner in the $z$ direction towards the cell. The deflection of the cantilever is detected by a photodiode when the tip comes in contact with the cell. The difference between the cantilever movement $z$, and the cantilever deflection $d$, represents the cell indentation $\delta=z-d$. The stiffness depends not only on Young's modulus but also on the geometry of the tipsurface contact. Therefore, the geometry and spring constant of the cantilever are calibrated in the same way for the live and fixed cells (see Fig.3). In the following experiments, we used a spherical tip (sphere diameter: $5 \mu \mathrm{m}$ ) with spring constants $0.006 \mathrm{~N} / \mathrm{m}$ and $1.75 \mathrm{~N} / \mathrm{m}$ for the live and fixed cells respectively( our experience, we found that the fixed cells were stiffer compared to the live cells).

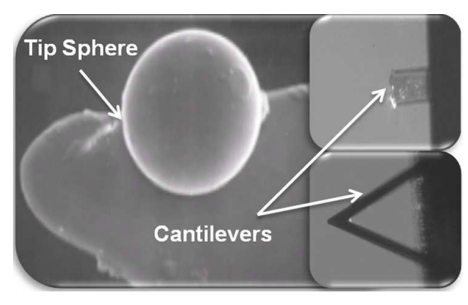

Fig. 3. Geometrical and spring constant Tip used for the live and fixed (mESC): The cantilevers attached with a spherical probe (5 $\mathrm{m}$ in diameter), the silicon nitride cantilever(Novascan Technologies, Inc).

\section{B. AFM calibration}

Before any experiment is done, it is necessary to perform the calibration of the cantilever to determine its resonant frequency. The resonant frequency measurement enables the user to determine it lies within the specified range provided by the manufacturer. If it does not, then it helps to determine if the tip has been damaged. The determination of spring constant, $K$, is a two step process:

- Determine the slope of contact region from a force curve to determine the sensitivity of the cantilever, and

- Measure the Thermal Power Spectral Density (PSD) to determine the resonant frequency of the cantilever and to confirm that the cantilever and light source are aligned. This confirms that the system is functioning properly. The results are plotted as the deflection amplitude data using Fourier transform in $\mathrm{m} / \mathrm{sqrt}(\mathrm{Hz})$ vs. the frequency in $\mathrm{Hz}$ (see Fig. 4). The spring constant of the cantilever was determined experimentally for each tip used in our studies using the IGOR software interface supplied by Asylum Research.

\section{Mouse Embryonic Stem Cells CHARACTERIZATION}

\section{A. Cell culture preparation}

It is recommended to use specially coated dishes or slides to facilitate adhesion. Such Petri dishes or slides are available commercially, cells should be attached to some rigid substrate, usually either a slide or the bottom of a Petri dish. In our study, the mouse embryonic stem cells 


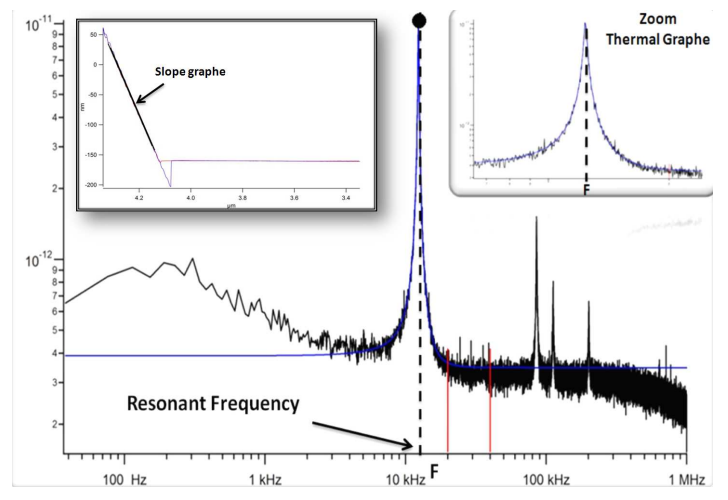

Fig. 4. Thermal Graph to determine the resonant frequency of the cantilever: the curve on left represents the slope of contact region from a force curve to determine the sensitivity of the cantilever and the curve on right represents the zoom-in view of the resonant frequency plot.

(mESC) R1 (SCRC-1011, American Type Culture Collection (ATCC), Manassas, VA) were grown on 0.1(percent) gelatin coated plates in the absence of feeder cells. The ES medium consisted of $1000 \mathrm{U} / \mathrm{ml}$ leukemia inhibitory factor (LIF, ESGRO, Chemicon, Temecula, CA), 15(percent) fetal bovine serum (FBS)(Invitrogen), and basic medium that included Knockout Dulbecco's modified Eagle's medium (Invitrogen), $2 \mathrm{mM}$ L-glutamine, 1x non-essential amino acids, and 0.1 $\mathrm{mM}$ mercaptoethanol. Differentiation was induced by removal of LIF from the medium. Prior to experiments, cells were dispersed using trypsin to obtain single cells and were plated on $60 \mathrm{~mm}$ tissue culture petri dish. Fixed mouse ES cells were obtained by treating the live mouse ES cells with 4 minutes and were stored in phosphate buffered saline (PBS).

\section{B. Cantilever interaction with the surface}

Prior to using the cantilever to measure the interaction force between the cell and the tip, it is important to observe the behavior of the cantilever, including instability and hysteresis resulting from adhesive forces. This measurement is called the Approach-Retract curve. Figure 5 represents the quantitative information of forces between the AFM probe tip and the sample as a function of the tip-sample distance.

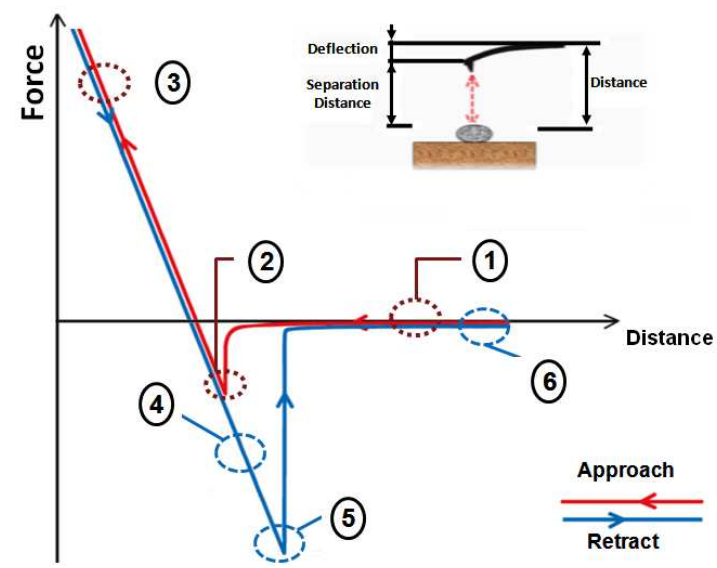

Fig. 5. Approach-Retract curve for a typical AFM cantilever interaction with the surface.

\section{Geometry of the cell}

The cells were 10 and $15 \mu \mathrm{m}$ in diameter. The phase contrast module enabled imaging low contrast, transparent cells in fluid via an inverted microscope (Model: TE2000U, Nikon, Inc) as shown in figure 4.25. The cells were stained with Hoescht 33342 and were visualized by fluorescence microscopy (FM) as shown in figure (Fig.6). The threedimensional geometry of mESC colony is visualized via the AFM contact mode imaging (Fig.8). The mESC colony size can be estimated through image analysis (ImagePro software from Asylum). To determine the cells height, two force indentation measurements are necessary, i.e, hard substrate and (ii) cell surface. First, initial height $h_{0}$ is measured by bringing the AFM tip in contact with the hard substrate and then bringing the AFM tip on the cell surface $h_{1}$. The cell's height is the difference $h=h_{0}-h_{1}$.

\section{Mechanical Characterization of MESC: REVIEW OF ANALYTICAL MODELS}

To estimate the mechanical properties of biological cells using the AFM, various analytical models can be used to estimate young's modulus of mESC in live as well as fixed cells, we need to perform mechanical nanoindentation tests.

\section{A. The Hertz contact}

The Hertz contact model has been used extensively by the AFM community to quantify the mechanical property of biological samples using AFM [8],[9]. The Hertz contact model describes the simple case of elastic deformation of two perfectly homogeneous smooth surfaces touching under load. By collecting force-distance curves on a time scale where viscous contributions are small, the forces measured are dominated by the elastic properties. In our work, the geometry of the tip used for all experiments is spherical. The mechanical interaction between the spherical tip and mESC can be described by the Hertz contact model of two elastic bodies [20]. The model assumes that:

- The material properties of the the tip and the cell are isotropic and homogeneous.

- The normal contact of the two bodies is adhesionless and frictionless.

- The contact geometry is assumed to be axisymmetric, smooth and continuous.

Furthermore, the theory has been experimentally shown to be valid at small deformations.

The relationship between the indentation $\delta$, and the loading force $F$ is given by:

$$
F=\frac{4}{3} E^{*} R^{\frac{1}{2}} \delta^{\frac{3}{2}}
$$

where $E^{*}, R$ are the combined modulus and the relative curvature of the tip and the cell respectively

$$
\begin{gathered}
\frac{1}{E^{*}}=\frac{1-\nu^{2}}{E}+\frac{1-\nu^{\prime 2}}{E^{\prime}} \\
\frac{1}{R}=\frac{1}{R_{1}}+\frac{1}{R_{2}}
\end{gathered}
$$



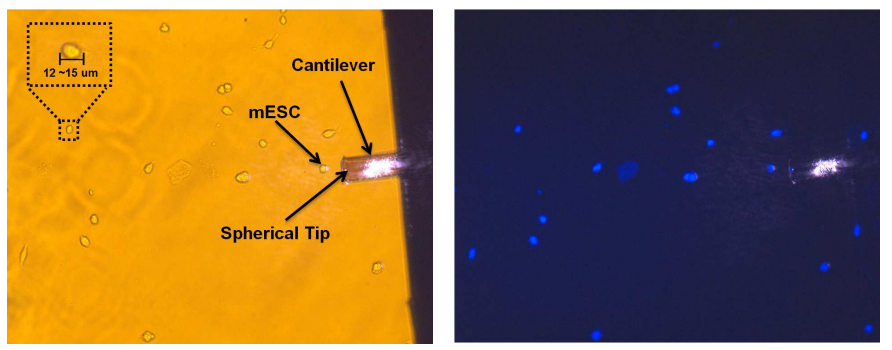

Fig. 6. Phase Contrast picture.

Fig. 7. Fluorescence image of mESC

where $\left(E, \nu, R_{2}\right)$ and $\left(E^{\prime}, \nu^{\prime}, R_{1}\right)$ represents the elastic modulus, Poisson's ratio, and the radius of the cell and cantilever respectively. The elastic modulus of the silicon nitride cantilever (used for live cells) and silicon cantilever (used for fixed cells) were 222.22 GPa and 168.17 GPa respectively. The elastic modulus of the cells is in range of $\mathrm{KPa}$ [11]. Hence, our assumption that the tip used for probing is infinitely stiff compared to the cell and Hertz contact model is valid. Thus, Eq. 1 can be rewritten as:

$$
F=\frac{4 E}{3\left(1-\nu^{2}\right)} R^{\frac{1}{2}} \delta^{\frac{3}{2}}
$$

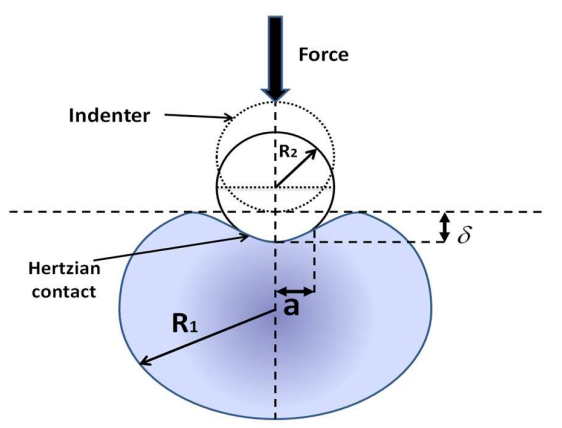

Fig. 9. Hertzian contact with a spherical tip: $F$ is the loading force and $\delta$ and $a$ are the indentation and contact radius respectively.

\section{B. The Capsule contact}

The second model considers the biological cell to be composed of a cell membrane and cytoplasm, is the capsule model [23]. The model assumes that the cell membrane is a thin film and that the inner cytoplasm provides a uniform hydrostatic pressure on the membrane (see Fig. 10). The model assumes the following:

- The cell membrane is linearly elastic.

- The deformation of the cell membrane is caused by stretching and the bending is neglected.

- The cell is free of initial membrane stress or residual stress.

- The cell volume is constant.

The force $(F)$, indentation $(\delta)$ relationship is given :

$$
F=\frac{2 \pi h R_{0}\left[1+R_{0} /\left(2 R_{s}\right)\right]^{2} E}{(1-\nu)\left(1+R_{0} / R_{s}\right)^{4}} \varepsilon^{3}+\frac{\pi}{2 \sqrt{2}} E h^{2} \sqrt{\varepsilon}
$$

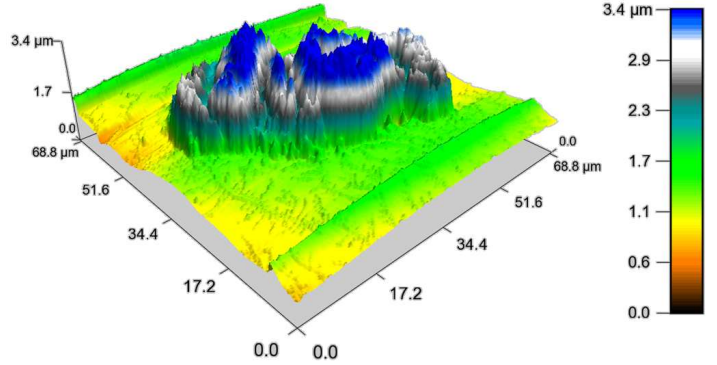

Fig. 8. Contact mode AFM (mESC colony).

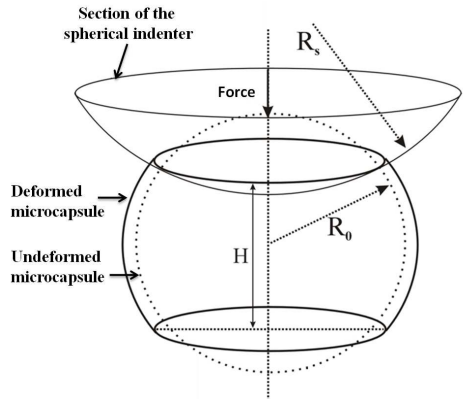

Fig. 10. Schematic of the microcapsule indented by a sphere with a loading force $F$.

where $E, \nu$, and $h$ represent the elastic modulus, Poisson's ration and the thickness of the capsule membrane respectively. $R_{0}, R_{s}$ is the radius of the capsule and the spherical indenter respectively. $\varepsilon$ is the relative deformation of the capsule given by $\varepsilon=1-\frac{H}{2 R_{0}}$ ( $H$ is the height of the capsule after indentation). The present model considers stretching as well as bending of the cell membrane, in our experiments the bending deformation term can be neglected for $\varepsilon>0.15$ [23].

\section{JKR and DMT contact}

The JKR and DMT contact models modify the Hertz theory by taken into account the adhesion between two elastic spheres. The model assumes that the adhesion force operates over a short distance within the contact region. According to this theory [25], contact radius $a$, indentation $\delta$ and pull-off force $P_{o f f}$ are represented by :

$$
\left\{\begin{array}{c}
a^{3}=\frac{3 R}{4 E^{*}}\left(F+3 \pi R w+\sqrt{6 \pi R w F+(3 \pi R w)^{2}}\right) \\
\delta=\frac{a^{2}}{R}-\sqrt{\frac{2 \pi w a}{E^{*}}} \\
P_{o f f}=\frac{3}{2} \pi R w
\end{array}\right.
$$

The DMT theory assumes the adhesion force operates just outside the contact zone where the surfaces are small distance apart. According to the DMT model, contact radius $a$, indentation $\delta$ and pull-off force $P_{\text {off }}$ are represented by the following equations:

$$
\left\{\begin{array}{c}
a^{3}=\frac{3 R}{4 E^{*}}(F+3 \pi R w+2 \pi R w) \\
\delta=\frac{a^{2}}{R} \\
P_{o f f}=2 \pi R w
\end{array}\right.
$$



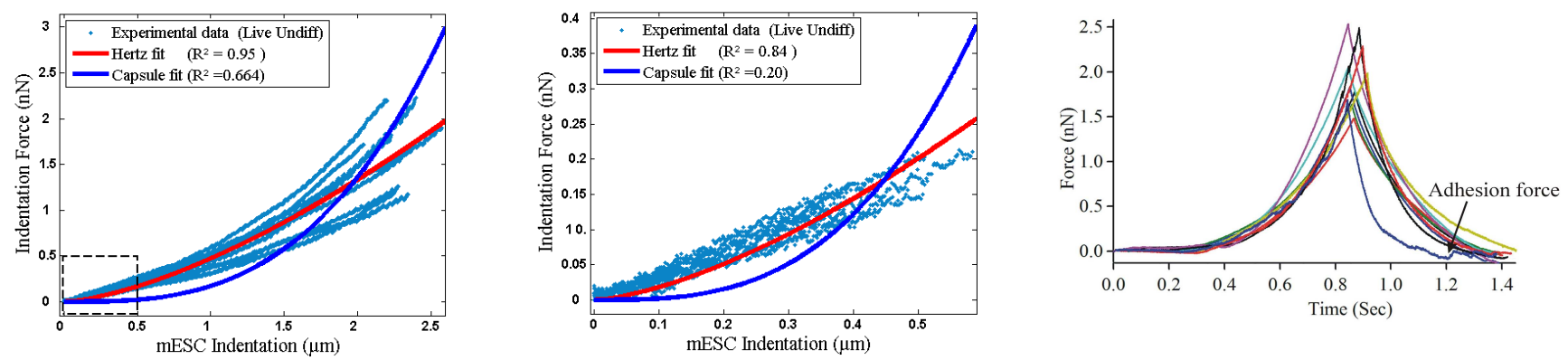

Fig. 11. Force versus indentation for live undiff Fig. 12. Force versus indentation for live undiff mESC. (small deformation).
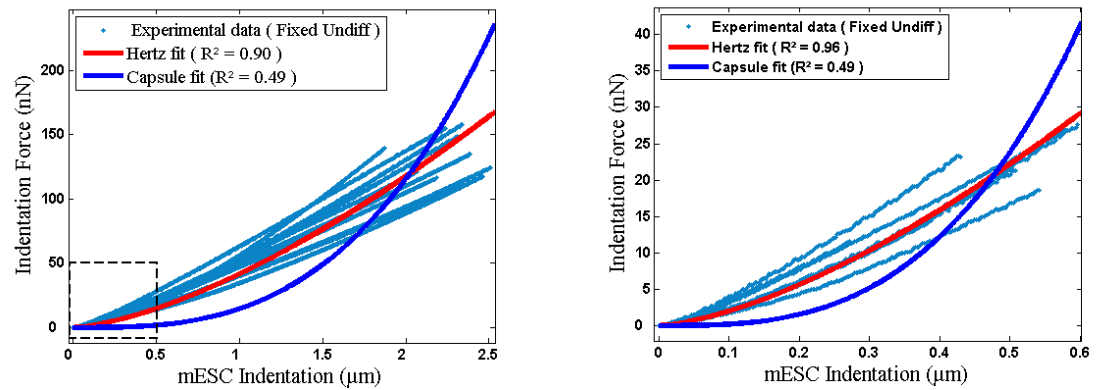

Fig. 13. Load-unload versus time for live undiff.

Fig. 14. Force versus indentation for fixed undiff Fig. 15. Force versus cell indentation for fixed Fig. $16 . \quad$ Load-unload versus time for fixed undiff. mESC. undiff(small deformation)

\section{Comparison between experimental data and analytical models}

We performed single indentation studies with a spherical tip on $20 \mathrm{mESC}$; 10 fixed and 10 live cells with spherical tip. We evaluate and compare between the different analytical models to determine whether it appropriately predicts the force-indentation relationship of mESC. We also present the force (loading and unloading) versus time profiles and determine whether force of adhesion exists between the tip and the cells. The figures (Fig.11, Fig.14) show the force versus cell indentation for live and fixed undifferentiated mESC using the capsule and the Hertz model respectively with corresponding $R^{2}$ value. Based on the experimental data, we performed a least square fit on the data set and the corresponding $R^{2}$ value was found to be 0.91 and 0.98 for the fixed and live undifferentiated mESC respectively. The figures (Fig.13, Fig.16) present the force (loading and unloading) versus time of fixed and live undifferentiated mESC, in our experiments, we observed that the adhesion force exists for only one of live undifferentiated but does not exist for the fixed cells. This could be due to the wear of the spherical tip [25] as there are no adhesion forces. The equation(4) was used to determine the global elastic modulus of the mESC. The average elastic modulus was $17.87 \mathrm{KPa}$ and $0.217 \mathrm{KPa}$ for the fixed and live undiff respectively (see Fig.17). The standard deviation for both of them was $3.37 \mathrm{KPa}$ and $0.05 \mathrm{KPa}$ for the fixed and live undiff cells. Finally, for our experiments, we infer that Hertz model appropriately describes the mechanical behavior of the live and fixed undifferentiated mESC.
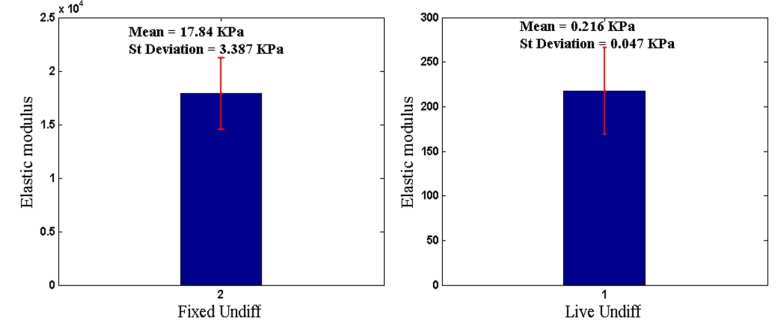

Fig. 17. Elastic modulus for live and fixed undifferentiated mESC calculated from the Hertz contact model for the small deformation (range $0.5 \mu \mathrm{m}$ ) with the error bar.

FEM simulations may help us to answer the unsolved question: in which indentation range can we use the Hertz model to determine the cell global equivalent Young modulus since the Hertz theory was shown to be valid at small deformations ?

\section{Finite Element Model (FEM) Simulation of CELL INDENTATION RESULTS}

\section{A. Finite Element Simulations of spherical tip indentation for live and fixed $m E S C$}

The Fig.18 presents our approach for deducing the Young's modulus from spherical-indentation tests. In a first time, the geometrical characteristics and the mechanical properties for different cells are determined with AFM indentation and analytical Hertz contact model. These properties are injected in the finite element simulation. The indentation depth versus force indentation are measured using the AFM 
and compared to the FEM predicted results. The finite element simulations are performed using an industrial research and development finite element code Cast $3 \mathrm{M}^{1}$. The FE model of the mESC is based on the following hypothesis:

- The mechanical problem is axisymmetric.

- The spherical tip is undeformable.

- the cell is supposed to have homogeneous, isotropic (visco)hyperelastic and nearly incompressible material properties.

The assumption of homogeneity, which may seem to be wrong, implies that we study a global equivalent elastic model of the cell. Furthermore, as already mentioned, the microsphere AFM-indentation test averages the contribution of multiple cytoskeleton fibres and smoothes the area contact geometry as needed for the use of the Hertz contact model. We used the Saint-Venant Kirchhoff hyperelastic model for which the elastic potential is given by:

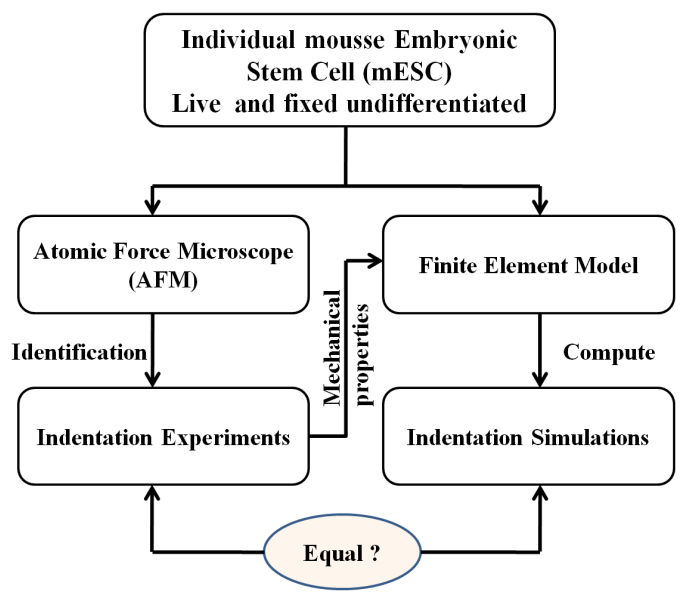

Fig. 18. Approach for validating finite element computational models. First, the mechanical properties and geometric parameters are determined with AFM indentation experiments before to be injected in the FEM. Then, the indentation versus force indentation are measured using the AFM and compared to the models predicted results.

$$
W(\mathbf{E})=\frac{\lambda}{2}(\operatorname{tr} \mathbf{E})^{2}+\mu\left(\operatorname{tr} \mathbf{E}^{2}\right)
$$

Since the strain energy function $W$ is quadratic in terms of strain invariants, we obtain a linear relation between the second Piola-Kirchhoff stress tensor and the Green-Lagrange strain tensor and it is given by:

$$
\begin{aligned}
& \mathbf{S}=\lambda(\operatorname{tr} \mathbf{E}) \mathbf{I}+2 \mu \mathbf{E} \\
& \text { or } S_{i j}=\lambda E_{k k} \delta_{i j}+2 \mu E_{i j}
\end{aligned}
$$

The Saint-Venant Kirchhoff law extends the Hooke's law for large deformations. The main difference is that the stress field, which was a linear function of the gradient of the displacement field $\operatorname{grad} \underline{U}$, is now a quadratic function of $\operatorname{grad} \underline{U}$. Since a rate sensitivity effect and hysteresis may be observed in the force vs. indentation loading-unloading

\footnotetext{
${ }^{1}$ The FE code Cast3M is developed by the Department of Mechanics and Technology (DMT) of the French Atomic Energy Agency (CEA DEN/DM2S/SEMT)
}

curves, a first attempt was made to describe this viscoelastic dissipative behavior with a classical Kelvin-Voigt viscoelastic model. The relation between the second Piola-Kirchhoff stress tensor, the Green-Lagrange strain tensor and the the rate effect leads to:

$$
\begin{gathered}
\mathbf{S}=(\lambda(t r \mathbf{E}) \mathbf{I}+2 \mu \mathbf{E})+\eta(\lambda(t r \dot{\mathbf{E}}) \mathbf{I}+2 \mu \dot{\mathbf{E}}) \\
S_{i j}=\left(\lambda E_{k k} \delta_{i j}+2 \mu E_{i j}\right)+\eta\left(\lambda \dot{E}_{k k} \delta_{i j}+2 \mu \dot{E}_{i j}\right) \\
\text { TABLE I }
\end{gathered}
$$

MECHANICAL AND GEOMETRICAL PROPERTIES USED IN THE FEM

\begin{tabular}{|c||c|c||c|c|}
\hline \multicolumn{1}{|c||}{} & \multicolumn{1}{|c||}{ Fixed Undiff } & Live Undiff \\
\hline \hline Diameter of the cell $(\mu \mathbf{m})$ & \multicolumn{2}{|c||}{12} & \multicolumn{2}{|c|}{10} \\
\hline height of the cell $(\mu \mathbf{m})$ & \multicolumn{2}{|c||}{8} & \multicolumn{2}{|c|}{7.2} \\
\hline Elastic modulus $(\mathbf{K P a})$ & 23.5 & 26.7 & 0.15 & 0.169 \\
\hline Indentation range $(\mu \mathbf{m})$ & 2 & 0.5 & 2 & 0.5 \\
\hline Poisson ratio & \multicolumn{2}{|c||}{0.49} & \multicolumn{2}{|c|}{0.49} \\
\hline Diameter of the Tip $(\mu \mathbf{m})$ & \multicolumn{2}{|c|}{5} & \multicolumn{2}{|c|}{5} \\
\hline
\end{tabular}

The geometrical characteristics were obtained from experimental data, namely the diameter and height of the cell(Tab.I). The main difficulty of identifying material properties from the non linear force-displacement curve is that this geometrical non-linearity can come from both large strains and contact non-linearities. Indeed, the cells are indented $\approx 2 \mu \mathrm{m}$ which is not infinitesimal compared to the height of the cells $\approx 8 \mu \mathrm{m}$. If the non-linearity is attributed only to the contact geometry, the Young's modulus values deduced from the classical Hertz equations may be unreliable[26]. A classical solution, but computationally very expensive, is to perform an inverse finite element solution to determine the optimum values of (visco)hyperelastic material properties. We chose a different strategy, computationally more efficient: we limited the fitting to data points that do not exceed $0.5 \mu \mathrm{m}(\approx 5 \%$ of relative nominal strain). The viscosity coefficient was roughly estimated by successive simulations.

Numerical FEM used two structure meshing; first the coarse mesh size composed of 398 nodes and 724 triangles and second with 1628 nodes and 3108 triangles(Fig.20). The results of mESC deformations are visualized in (Fig.21) and (Fig.22) for a fixed mESC. The FEM analysis shows that moderate to large deformations are observed around the contact region $(\approx 20 \%$ for $0.34 \mu \mathrm{m}$ and $\approx 40 \%$ for $1 \mu \mathrm{m})$. As a validation, the Hertz contact model and FEM simulations have been compared with force-displacement curves obtained from AFM indentation experiments on both undifferentiated fixed (Fig.24) and live undifferentiated (Fig.23) single mESC. These comparisons show clearly that the Hertz contact model cannot be used for the whole indentation range:

- if the Young modulus is deduced from the full datasets, the finite element simulation show good agreement to experimental results but only until moderate deformations $(<\approx 10 \%)$.

- if the Young modulus is deduced from a truncated dataset, the finite element simulation show good agree- 


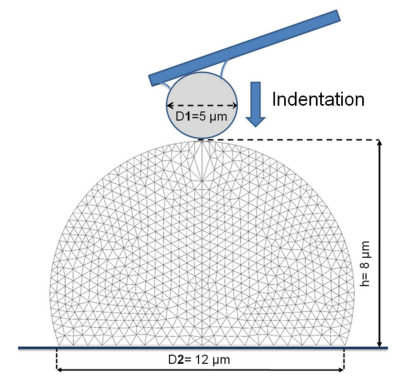

Fig. 19. Mesh geometry of the cell.

ment to experimental results for the whole simulation and differ significantly from Hertz contact model for large deformations $(>\approx 10 \%)$.

Finally, hysteresis was quantified experimentally and numerically by subtracting the area under indentation and retraction curves, which represents the viscous dissipation of energy into the cell. The FEM simulations and experimental data are shown in Fig.25. Calculated in this manner, hysteresis was about $15-20$ percent, indicating that energy dissipation due to the material viscosity contribution is low at this probe velocity and force measurements are dominated by the cell elastic behavior.

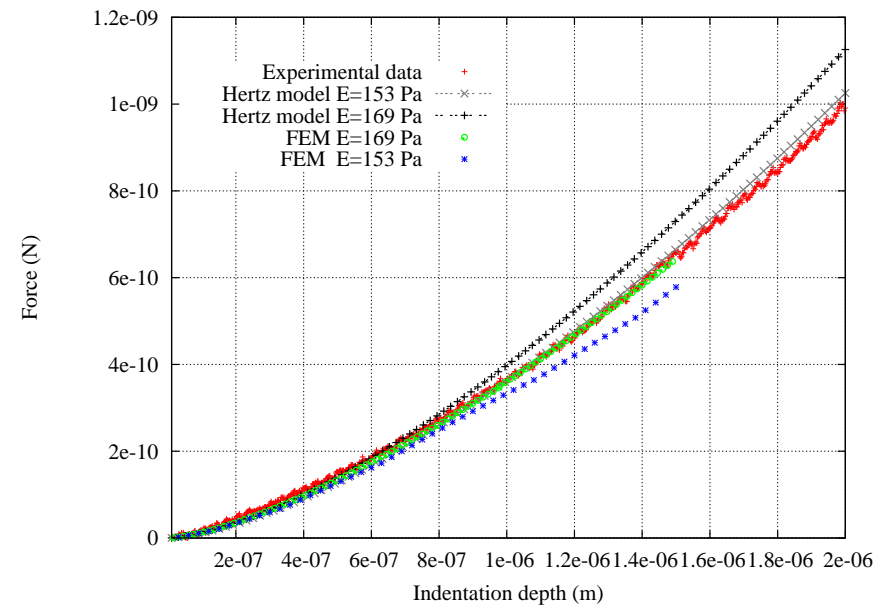

Fig. 22. Force versus indentation for live undiff cells: Comparison between the FEM simulation(Indentation range large and small) and experimental data.

\section{B. PHANToM Haptic Device}

In order to provide force feedback to the user during the cell contact, the AFM is integrated with the PHANToM device. The deflection channel of the AFM controller was connected to a data acquisition board (model:DS1103, dSPACE Inc, Wixom, MI). The force exerted on the cell and hence transmitted to the haptic feedback device is given by:

$$
F=K d
$$

Where $K$ is the spring constant of the cantilever and $d$ the cantilever deflection Thus, our haptics-enabled AFM system obtains the relationship between the force exerted on the cell

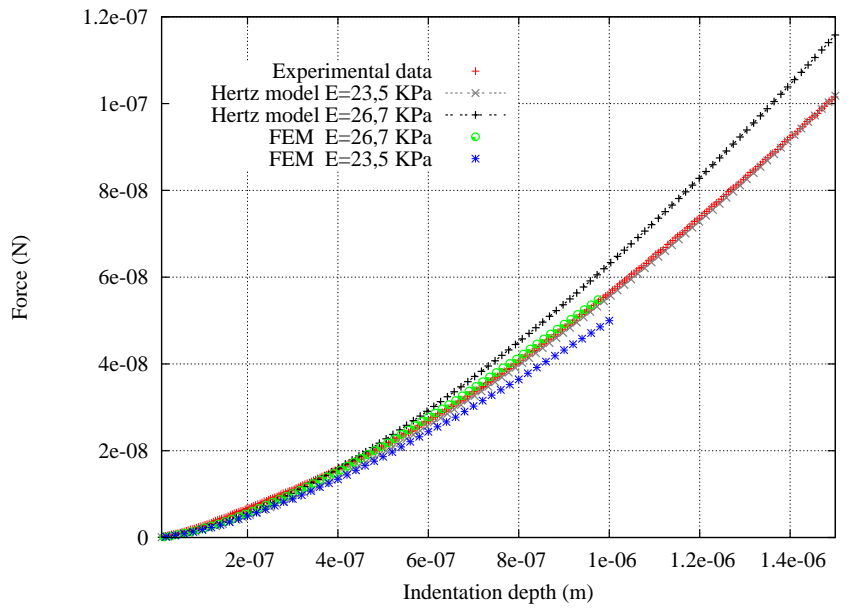

Fig. 23. Force versus indentation for fixed undiff cells: Comparison between the FEM simulation(Indentation range large and small) and experimental data.

and the corresponding deformation. The force detected by the AFM during cell contact is acquired by a data acquisition board in real-time (model: dSPACE DS1103). The interface allowed the user to feel the cell indentation force in real time. The force was amplified by a factor of $10^{7}$ for the human operator to perceive the change in force during cell indentation by the AFM cantilever between the fixed and live cells. This tool can be used as a mechanical marker to characterize the biological state of the cell. The haptic feedback was rendered in real time to the operator during cell indentation by the AFM tip. The operator was able to feel the change in the stiffness during cell deformation between the fixed and live cells..

\section{Analysis and Discussion}

Despite variations between cells of the same population, the experimental data show that fixed cells are more stiffer than live cells $\left(10^{2}\right.$ to $10^{3}$ magnitude of order). Nevertheless, fixed and live mESC present common mechanical nonlinear responses. The finite element simulations show good agreement with both Hertz contact model and experimental indentation for low to moderate deformations (around 10\% of relative nominal strain (see Fig.22, Fig.23). Furthermore, as expected, the finite element simulations show perfect adequation with Hertz contact model for low deformation. The differences between our experimental results and nu- 
merical simulations for large deformations can be explained by the fact that Hertz contact model is only valid for low deformations ([21], [22]). A first attempt has been made to approach observed experimental hysteresis with a simple Kelvin-Voigt model. Preliminary simulations show quantitatively good agreement with experimental data (Fig. 24).

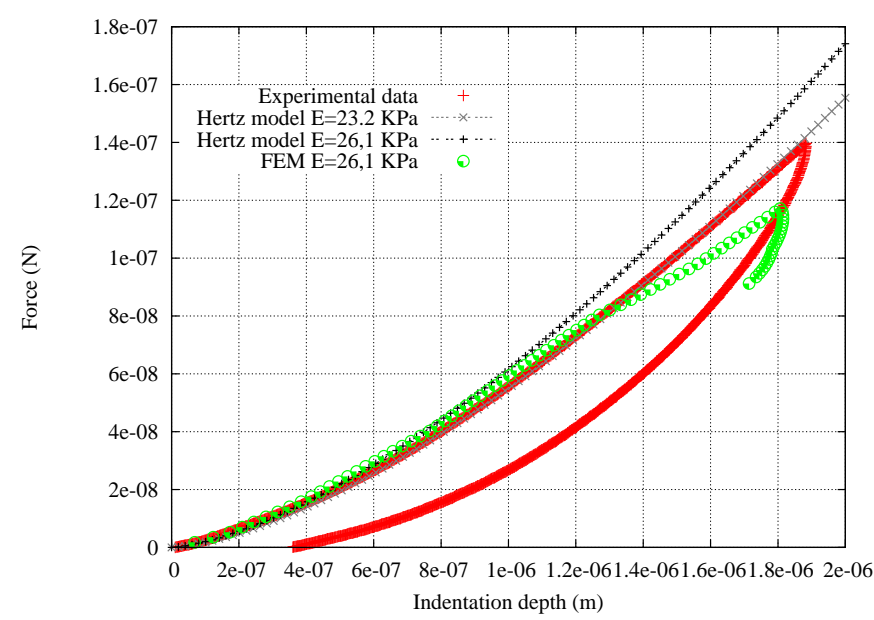

Fig. 24. Experimental viscoelastic hysteresis and finite element simulation.

\section{CONClusions And Future WORK}

Atomic force microscopy has rapidly become a valuable tool for quantifying the biophysical properties of single cells. We have presented the mechanical characterization of single mESC indentation-relaxation studies using microspheremodified AFM probes for different fixed and live undifferentiated mESCs. As the interpretation of atomic force microscopy-based indentation tests is highly dependent on the use of an appropriate contact model, we have used Hertz contact model based on our recent work on analyzing the various contact models appropriate for the AFM tip and mESC interaction. Finite element simulations (FEM) of spherical tip indentation proves the effectiveness of computational model to predict the mESC deformation behavior during mESC indentation and relaxation tests. As a perspective, the proposed FEM model constitutes a basis for simulation of nanoinjection tasks using virtual haptic feedback model for tissue engineering applications and development of new therapies for regenerative medicine. In our ongoing and future work, we are developing a real-time force-feedback simulator dedicated to AFM indentation for various living cells. To achieve these goals, the proposed finite element model (FEM) takes into account the nonlinear elasticity cell model, the geometry and properties of AFM-tip indenters (spherical and pyramidal probes).

\section{REFERENCES}

[1] J. Settleman, Tension precedes commitment-even for a stem cell. Mol Cell, vol.14, pp. 148-150, 2004.

[2] V.E., Meyers, M. Zayzafoon, J.T. Douglas, and J.M. McDonald, RhoA and cytoskeletal disruption mediate reduced osteoblastogenesis and enhanced adipogenesis of human mesenchymal stem cells in modeled microgravity, J. Bone Miner. Res. vol.20, pp.1858-1866, 2005.
[3] R. McBeath, D.M. Pirone, C.M Nelson, K. Bhadriraju, and C.S. Chen, Cell shape, cytoskeletal tension, and RhoA regulate stem cell lineage commitment. Dev. Cell. vol. 6, pp.483-495, 2004.

[4] J.Desai, A.Pillarisetti, A. D.Brooks, Engineering Approaches to Biomanipulation, Annual Review of Biomedical Engineering, 9, pp. 35-53, 2007.

[5] F. Arai, D. Ando, T. Fukuda, Y. Nonoda, T. Oota, Micromanipulation based on micro physics, strategy based on attractive force reduction and stress measurement, in Proc. IEEE Int. Conf. Robotics Automation, pp. 236-241, 1995.

[6] Bausch AR, Moller W, and Sackmann E., Measurement of local viscoelasticity and forces in living cells by magnetic tweezers, Biophys Journal, vol.76, pp.573-579, 1999.

[7] T. Ohasti, M. Hagiwara, D. L. Bader, N. M. Knight, Intracellular mechanics and mechanotransduction associated with chondrocyte deformation during pipette aspiration, Biorheology, vol. 43, no3-4, pp. 201-214, 2006.

[8] J. L. Alonso and W. H. Goldmann, Feeling the forces: atmoic force microscopy in cell biology, Life Sciences, vol. 722553 - 2560, 2003.

[9] A. Touhami, B. Nysten, and Y. F. Dufrene, Nanoscale mapping of the elasticity of microbial cells by atomic force microscopy, Langmuir, vol. 19 pp 4539 - 4543, 2003.

[10] Darling, E.M., S. Zauscher, and F. Guilak, Viscoelastic properties of zonal articular chondrocytes measured by atomic force microscopy, Osteoarthritis Cartilage, 14:571-579, 2006.

[11] Mahaffy, R.E., S. Park, E. Gerde, J. Kas, and C.K. Shih. Quantitative analysis of the viscoelastic properties of thin regions of fibroblasts using atomic force microscopy, Biophys. J. 86:1777-1793, 2004.

[12] Alcaraz, J., L. Buscemi, M. Grabulosa, X. Trepat, B. Fabry, R. Farre, and D. Navajas, Microrheology of human lung epithelial cells measured by atomic force microscopy, Biophys. J. 84:2071-2079, 2004.

[13] A. Pillarisetti, C. Keefer, J. P. Desai, Mechanical Response of embryonic stem cells using haptics-enabled atomic force microscopy, in International Sympoisum on Experimental Robotics, Athens, Greece, 2008

[14] Robert E. Rudd, Michael McElfresh, Eveline Baesu, Rod Balhorn, Michael Allen and James Belak, Modeling of the Mechanical Deformation of Living Cells in Atomic force Microscopy, Biophysical Journal, 2007.

[15] Valentin Lulevich, Tiffany Zink, Huan-Yuan Chen, Fu-Tong Liu, and Gang-yu Liu, Cell Mechanics using Atomic Force Microscopy-based Single-Cell Compression, Biophysical Journal, 2007.

[16] Robert Tatyana G. Kuznetsova, Maria N. Starodubtseva, Nicolai I. Yegorenkov, Sergey A. Chizhik and Renat I. Zhdanov, Atomic force Microscopy probing of Cell Elasticity, Biophysical Journal, 2007.

[17] Robert E. Rudd, Michael McElfresh, Eveline Baesu, Rod Balhorn, Michael Allen and James Belak, Modeling of the Mechanical Deformation of Living Cells in Atomic force Microscopy, Biophysical Journal, 2007.

[18] H.Ladjal, J-C.Hanus, A.Ferreira, Interactive Cell Injection Simulation based on Biomechanical Tensegrity Model, IEEE International Conference on Robots and Intelligent Systems, Nice, France, 2008.

[19] M. Falvo and R. Superfine, Mechanics and friction at the nanometer scale, J. Nanoparticle Res., vol. 2, pp. 237-248, 2000.

[20] A. C. Fischer-Cripps, Introduction to contact mechanics, Second ed. New York: Springer, 2007.

[21] Y. Tatara, On Compression of Rubber Elastic Sphere Over a Large Range of Displacements - Part 1: Theoretical Study, J. Eng. Mater. Technol. 113, pp. 285-291, 1991.

[22] K.K. Liu, D. R. Williams and B.J. Briscoe The large deformation of a single micro-elastomeric sphere, Journal of Physics D: Applied Physics, Vol. 31 (3), pp. 294-303, 1998.

[23] V. V. Lulevich, D. Andrienko, and O. I. Vinogradova, Elasticity of polyelectrolyte multilayer microcapsules, Journal of Chemical Physics, vol. $120(8)$, pp. 3822 - 3826, 2004.

[24] E. Kwon, Y. Kim, J. Park, D. Kim, and H. Jung, Measurement of pulloff forces by atomic force microscope in liquids used for biological applications, Experimental Techniques 40-45, 2007.

[25] D. Maugis, Contact, Adhesion and Rupture of Elastic Solids: Springer, 2000 .

[26] Costa KD, Yin FCP, Analysis of indentation: implications for measuring mechanical properties with atomic force microscopy. ASME J Biomech Eng, 121:462-471, 1999. 